\title{
AGN VARIABILITY AND VLBI
}

\author{
L. B. BÅTH \\ Onsala Space Observatory \\ $S$-49992 Onsala \\ Sweden
}

25 November 1993

\begin{abstract}
This contribution discusses the connection between variability in radio and optical with structural variations observed with VLBI. Structural changes do not have to start in the core, and intensity variations may be caused by components in the jet outside the core. The scenario is probably more complicated than present day theories assume.
\end{abstract}

Key words: Active Galactic Nuclei, Quasars, Very Long Baseline Interferometry

\section{Introduction}

The activity in Active Galactic Nuclei is manifested in the grand radio jets we can observe in some source, e.g. Cyg A, and in the intensity variations over the whole electromagnetic spectrum. The intensity variations have been discussed previously at this symposium (Bregman), but let me remind you that the very first detection of such variablity was in CTA102 (Sholomitskii 1965). At that time VLBI was not available and the lower limit of the angular size of the source was believed to be 0 ".01. The size scale was calculated from the intensity variations by Sholomitskii to be $\approx 0.1 \mathrm{pc}$, resulting in a distance to the CTA102 of $\approx 2 \mathrm{Mpc}$. Later it was shown that CTA102 has a redshift of $z=1.037$, but theories developed since then have clearly shown that beaming would allow a seemingly contradiction of the Causality theorem.

The purpose of VLBI is to study the structural changes within the fine-scale strucure of radiojets. The purpose of this talk will be to discuss the connection between such structural changes and the intensity variations:

- do large intensity changes result in structural changes?

- do structural changes result in changes in intensity?

As a corollary we can also raise the following questions:

- do all intensity variations originate in the core?

- do all structural changes originate from the core?

\section{Where is our point of reference?}

In order to discuss structural changes in an object it is necessary to define a point of reference with regard to the rest of the universe. A fundamental question for our further discussion is thus where the socalled "core" is. This is in VLBI usually assumed to be the component at the end of the milliarcsecond jet which has a flat

181

T. J.-L. Courvoisier and A. Blecha: Multi-Wavelength Continuum Emission of AGN, 181-186.

(C) 1994 IAU. Printed in the Netherlands. 

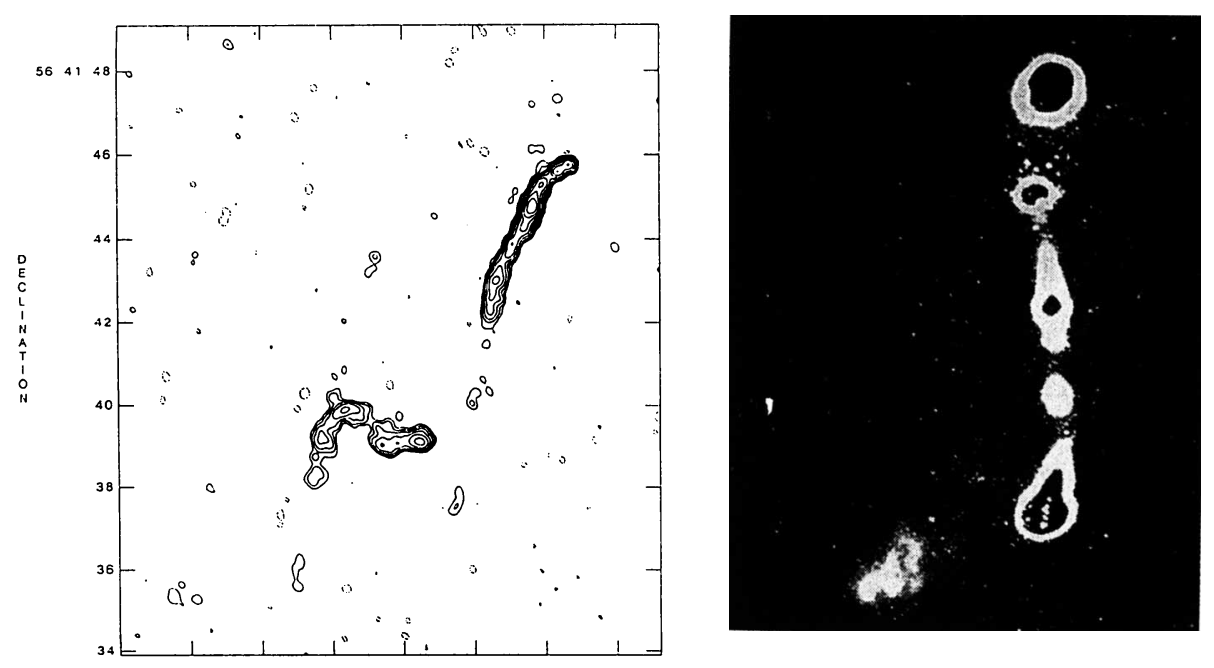

Fig. 1. Radio structure of $1857+566$ with VLA, courtesy of C.Akujor (left); $18 \mathrm{~cm}$ VLBI image of $1422+202$ (right)

spectrum between 5-10 GHz. It has been shown, however, that this component is not at the same position on the sky at all frequencies. Marcaide et al. (1985) showed e.g. that the core of $1038+525 \mathrm{~A}$ has a positionoffset on the sky of about 1 mas between 2 and $8 \mathrm{GHz}$. Therefore we may well assume that what we state to be the core is simply the first visible part of the jet, and that the structural motions we measure are differential motions between components within the jet.

There are further indications for this from mmVLBI. The spectrum of 3C273 can be divided up into several parts, with the quiet radiospectrum broken down into a cm-wavelength part, coming from the jet emission and seen with VLBI at $\mathrm{cm}$ wavelengths, leaving a radiocomponent which peaks at around $150-200 \mathrm{GHz}$ and which is observed with VLBI at $3 \mathrm{~mm}$ wavelength (Bååth 1992). This may well be the real "radiocore" which is practically invisible at $\mathrm{cm}$ wavelengths.

\section{Radio structure}

A significant part of the discussion about the nature of extragalactic radiosources is based on comparisons of the brightness of the large-scale structure, usually being the outer radio lobes, relative the brightness of the compact radio core. Such comparisons all assume that the radio jet is straight. Figure 1a shows one of many examples of a definitely curved radio jet, and we can from such images deduce that most radiojets are curved in three dimensions on some scale. This means that Doppler boosting effects may differ significantly between various parts of the jet and any comparison between lobes and core is meaningless if it does not take differential Doppler boosting into effect. 

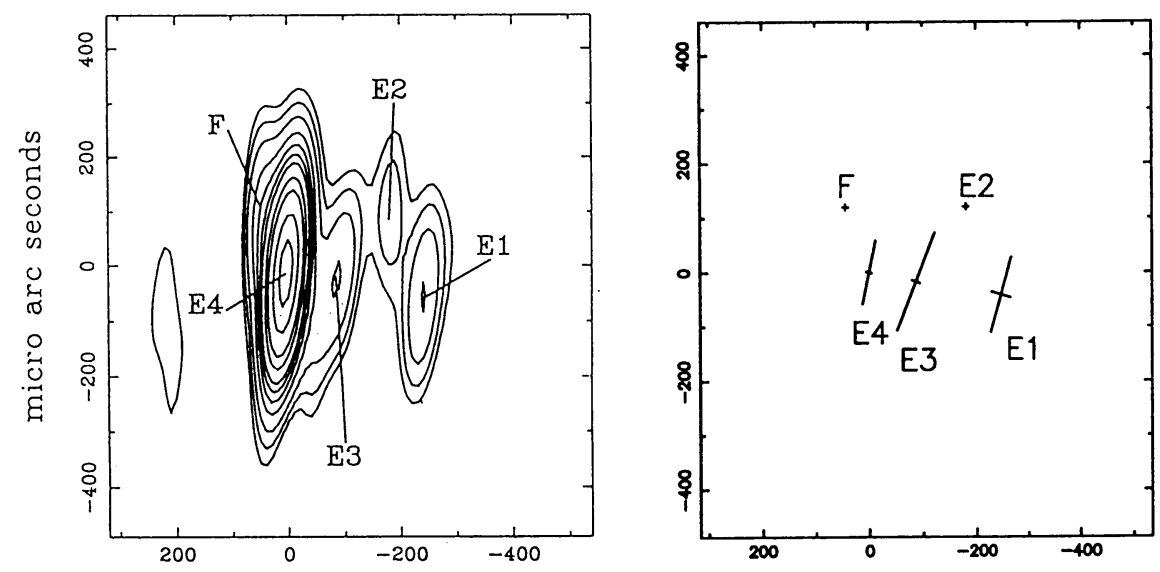

Fig. 2. Radio structure of $3 \mathrm{C} 273$ observed with $\lambda 3 \mathrm{~mm}$ VLBI: hybrid map (left), major and minor axes of deconvolved Gaussian fit (right)

Figure $1 \mathrm{~b}$ shows two images of the radio structure of the quasar $1422+202$. The large scale structure is from the VLA, while the overlayed fine scale structure was observed with VLBI at $\lambda 18 \mathrm{~cm}$. The VLBI observations were made with phasereferencing technique and the two images can therefore be correctly overlayed on the sky. This comparison of images show that the radiojet contains fine scale structure all the way out into the southern radiolobe. and that the radio jet is curved when studied on the milliarcsecond scale level, even though it looks straight with the lower resolution of the VLA. This is another indication that differential Doppler boosting over the radio jet may cause significant misinterpretation on any lobe/core ratio.

When discussing structure, and especially when we compare structure observed with different instrument, it is important to realize the vast different in scale we may observe. Images observed with the VLA can have resolution about 3", with $\mathrm{cm}$ wavelength VLBI about 3-1 mas, while mmVLBI may reach $40 \mu$ as: ranging over five magnitudes in resolution! A truely amazing dynamic, resulting in a very powerful arsenal of observing instruments. The physics over such a large variation in size scales may differ though, and we may not be able to apply the same discussion on the structure of the lobes as we do on the core.

\section{Intensity variations vs. structural variations}

One of the best examples of a close relationship between flux outbursts and birth of new VLBI components is BLLac. Mutel et al. (1990) have shown that a series of outbursts in BLLac can be identified with new components emerging from the core. There are a number of other examples of this phenomenon. We managed in 1988 to observe 3C273 (Bååth et al. 1991) with $\lambda 3 \mathrm{~mm}$ VLBI only about 60 days 
after a major outburst. The resulting hybrid map was dominated by a very bright component which could be identified with the outburst by subtracting the quiescent spectrum from the current radio spectrum, leaving a synchrotron spectrum with a $\lambda 3 \mathrm{~mm}$ flux density that fitted the VLBI component very well. A fit of gaussian components showed that the deconvolved component (Fig. 2) was thin along the jet and extended across the jet at a ratio of about 10:1, in good agreement with the burst-model by Marscher and Gear (1985).

The BLLac objects are of course of special interest for this discussion since one of their defining characteristics is the high degree of variability. The object $1749+701$ (Bååth and Zhang 1992) is especially interesting here as a good counter-example to BLLac. We did find this source to be superluminal, with components moving from the core with apparent superluminal speed. Knowing this speed it is then possible to deduce the probable time of birth of the component. We could not find any significant increase in flux density at any time close to this, but in all cases we did find that the birth seemed to be associated with a flip in polarization angle. This is also in accordance with the thin shock model, and the lack of a flux outburst can be explained if the geometry in this source is such that the Doppler boosting effect will not produce any enhancement of the radioation from the matter moving behind the shock front. There can be several explanations for this:

- The geometries for maximum Doppler boosting and maximal apparent speed are different.

- The structural speed does not have to be the same as the speed of the flow.

The latter point is a valid possibility, especially if the pattern, or structural, speed is caused by phase motion as suggested by Hardee (1989). Hardee suggests that the components are formed at the interception point between two modes of a reflective Kelvin-Helmholz instability within a flow of plasma through a galactic/intergalactic medium of a different density. We have found (Rantakyrö et al. 1993 ) that the jet in $3 \mathrm{C} 345$ is confined up to a point about 20 mas from the core where it starts to expand in a seemingly adiabatical way. Our explanation to this is that this point corresponds to where we expect the jet to enter the narrow line region and thus enter a region of less density where it can expand more freely. Therefore we do suspect that the density of the surrounding medium will indeed have an effect on the jet expansion and its internal structure.

The lesson we can draw from this is that VLBI components do not necessarily have to be born at a time of a flux outburst, but there will probably have been a change in polarization angle at that time.

\section{Do all changes originate from the core?}

$0735+178$ is a good example of that flux outbursts do not have to originate in the core (Baath, Zhang and Chu 1991). Our VLBI studies of this source showed for many years two components which were not moving relative each other, but with a third component moving between the two. We could from this motion predict 

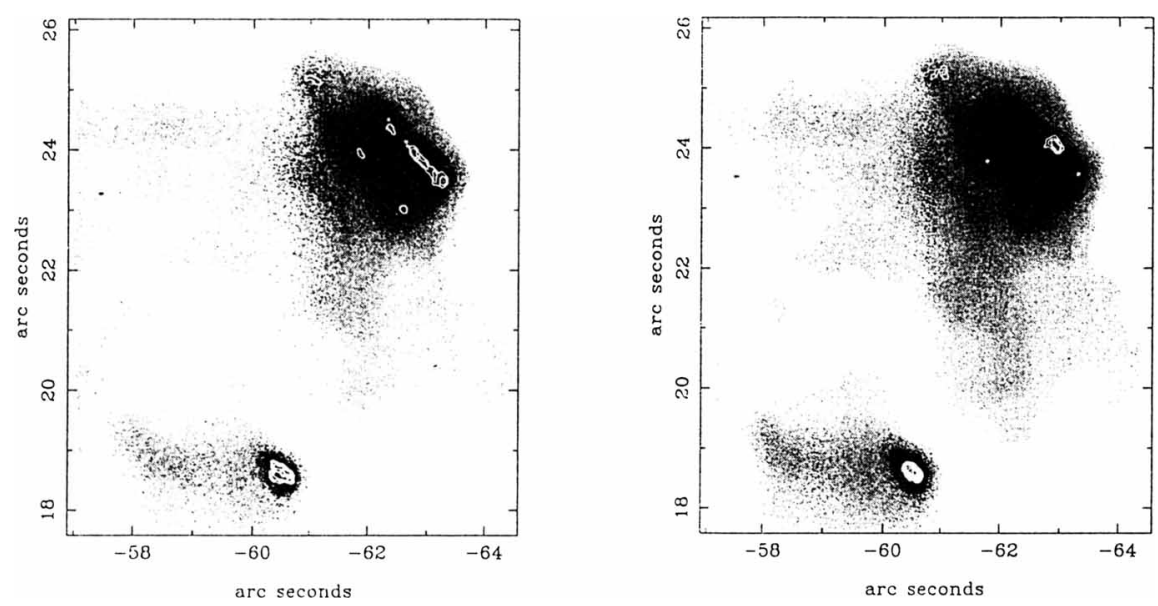

Fig. 3. The northern lobe of Cygnus A observed with the VLA (gray-scale) and MERLIN in 1983 (left) and 1993 (right)

when the moving component would reach the stable one, which took place at around 1990. At this time we observed the source with VLBI at $\lambda 1.3 \mathrm{~cm}$, and clearly the outer, stable, component showed up as a large, but bright, component, even though it had been invisible some years earlier at this wavelength. At the same time, the flux density of the source had steadily increased for some years, and the moving component had slowed down its apparent motion. We interpreted this as a source scenario where the jat starts at an angle of about $10^{\circ}$ to the line of sight and then curves so that at the point of the outer, stable, component the flow is directed directly towards the observer. This change in viewing angle would explain both the slowing down of the component, since the geometry would be less favourable, and the flux increase. Such phenomenon could very well happen in any source with a curved jet, and therefore conclusions from statistics may not be very trustworthy as long as we do not know the physics and the detailed structure of the plasma flow within the jet.

For many years we have neglected to look for any structural motions within the radio lobes of large radio sources, e.g. CygA. The reason for this neglect is mainly caused by that programme committees usually prefer more safe projects, and the general impression has been that no motion should be seen at sucg large distance from the core. MERLIN gave us time for studying Cygnus A at two epochs 10 years apart, 1983 and 1993. The source is too large for conventional mapping techinques, and the observations were made in spectral line mode, where the observing band was divided up into a number of narrow frequency channels. This procedure was first tested on a 5C12-field (Okopi and Båăth 1991) and then used to map the full area covering Cygnus $A$ with the full resolution of MERLIN at $6 \mathrm{~cm}$. The resulting 
image contains about 4000 pixel $^{2}$, and is using the VLA position of the core as the phase reference point on the sky. Our two epochs maps are shown in Figure 3 , overlayed on a gray scale VLA map at $\lambda 6 \mathrm{~cm}$ by Perley and collaborators (e.g. Dreher, Carilli, and Perley 1987). The VLA map was observed only a few days in time from our MERLIN observations in 1983, and there is indeed a very good correspondence between features. Our image from 1993 clearly indicates structural changes. The primary hotspot remains at the same place, but the north-west thin elongated feature has moved outwards. The apparent motion is about 70c, which indeed is superluminal. I do not, however, want to claim that this features started from the core and has moved all that way at a speed very close to the speed of light. A more reasonable suggestion is that this feature is a coherent structure caused by the turbulence which must be very important in the lobe, as well as within the jet itself. Such coherent structure is mainly a phase motion and can move in all three dimensions. It is therefore not very surprising if some of such structures will move in a direction which is very favourable to create apparent superluminal motion. I want to stress here that if such motion can happen in the lobes, then it can also happen in the jet which is believed to be highly turbulent. It would be very interesting to see some jet simulations where also turbulence is allowed to be an important factor.

Finally I would like to remind you that structural changes and intensity variations may arise in the medium between the source and us. Such changes may be created by gravitational lensing, especially by micro-lensing caused by star or starclusters in an intermediate galaxy. An effect which should be more common is refractive interstellar scattering (e.g. Rickett 1986), especially at long wavelengths.

\section{References}

Bååth,L.B., Padin,S., Woody,D., Rogers, A.E.E., Wright, M.C.H., Zensus,A., Kus, A.J., Backer, D.C., Booth, R.S., Carlstrom, J.E., Dickman, R.L., Emerson, D.T., Hirabayashi, H., Hodges, M.W., Inoue, M., Moran, J.M., Morimoto, M., Payne, J., Plambeck, R.L., Predmore, C.R., and Rönnanäng, B.: 1991, Astron.Astroph., 241, L1

Bååth,L.B., Zhang,F.J., and Chu,H.S.:1991, Astron.Astroph., 250, 50

Bååth,L.B.: 1992, Variability of Blazars, eds. Valtaoja,E. and Valtonen,M. (Cambridge Univ. Press, Cambridge), 229

Bååth,L.B. and Zhang,F.J.: 1992, Astron.Astroph.,262, 1

Dreher,J.W., Carilli,C.L., and Perley,R.A.: 1987, Astroph.J., 316, 611

Hardee,P.E.: 1989, Parsec-scale Radio Jets, eds. Zensus, J.A. and Pearson, T.J. (Cambridge Univ. Press, Cambridge), 266

Marscher,A. and Gear,W.: 1985, Astrophys.J., 298, 114

Mutel,R.L., Phillips,R.B., Bumai Su, and Bucciferro,R.R.: 1990, Astroph.J., 352, 81

Okopi,J. and Bååth,L.B.: 1991, Radio Interferometry: Theory, Techniques, and Applications, eds. Cornwell, T.J. and Perley, R.A. (Astron. Soc. Pac. Conf. Series), 253

Ricket,B.J.: 1986, Astroph.J., 307, 564 